\title{
Aula Aumentada y Aula Invertida, Los Nuevos Retos en Educación Superior Universitaria
}

\section{Augmented Classroom and Inverted Classroom, The New Challenges in Higher University Education}

\author{
Nilo Chuquimbalqui-Maslucán \\ Universidad Nacional Autónoma de Huanta, Ayacucho, Perú \\ E-mail: ncmsist@gmail.com ORCID: https://orcid.org/0000-0002-0833-4705
}

Recepción: 15/06/2021 Aceptación: 30/06/2021 Publicación: 30/07/2021

\section{Resumen}

El aula aumentada y aula invertida en cualquier nivel educativo es inminente que requiere la implementación de actividades académicas en un sistema de gestión del aprendizaje (Moodle, Chamilo, Teams, Google Classroom o Canvas). Por lo que, la finalidad de esta investigación es conocer a profundidad sobre aula aumentada y aula invertida, desarrollado mediante la descripción y explicación de investigaciones de otros autores. Se obtuvo: Primero, el análisis de los resultados de su aplicación sobre las experiencias de aula invertida en el área de la salud, los docentes consideran que la interpretación del electrocardiograma es un tema complicado de aprender durante la realización de los diagnósticos clínicos. El aula invertida representa una excelente alternativa para mejorar el proceso de enseñanza aprendizaje en medicina; Segundo, las experiencias de aplicación de aula invertida donde aparece un efecto de aprendizaje de gran velocidad que se refleja en la resolución de ejercicios y adquisición de nuevas estrategias; Tercero, existe una mejora en cuanto a la asistencia de los estudiantes en una clase virtualizada; Cuarto, el clima productivo de aprendizaje en el aula invertida se correlaciona positivamente con las percepciones de aprendizaje en cirugía de la Universidad de la Sabana, Chía, Colombia. Se concluye que aula aumentada y aula invertida, así como conectivismo y ubicuidad, son los nuevos retos en educación universitaria que requiere uso y aplicación adecuada en la formación de nuevos profesionales que exige el mundo global.

\section{Palabras clave}

Aula aumentada, aula invertida, conectivismo y ubicuidad. 


\section{Abstract}

The augmented classroom and flipped classroom at any educational level is imminent that requires the implementation of academic activities in a learning management system (Moodle, Chamilo, Teams, Google Classroom or Canvas). Therefore, the purpose of this research is to know in depth about the augmented classroom and the flipped classroom, developed through the description and explanation of research by other authors. It was obtained: First, the analysis of the results of its application on flipped classroom experiences in the health area, teachers consider that the interpretation of the electrocardiogram is a complicated subject to learn during the realization of clinical diagnoses. The flipped classroom represents an excellent alternative to improve the teaching-learning process in medicine; Second, the flipped classroom application experiences where a high-speed learning effect appears that is reflected in the resolution of exercises and the acquisition of new strategies; Third, there is an improvement in student attendance in a virtualized classroom; Fourth, the productive learning climate in the flipped classroom is positively correlated with the perceptions of learning in surgery at the Universidad de la Sabana, Chía, Colombia. It is concluded that augmented classroom and inverted classroom, as well as connectivism and ubiquity, are the new challenges in university education that require proper use and application in the training of new professionals that the global world demands.

\section{Keywords}

Augmented classroom, flipped classroom, connectivism and ubiquity.

\section{Resumo}

A sala de aula aumentada e a sala invertida em qualquer nível educacional é iminente que requer a implementação de atividades acadêmicas em um sistema de gestão de aprendizagem (Moodle, Chamilo, Teams, Google Classroom ou Canvas). Portanto, o objetivo desta pesquisa é conhecer em profundidade a sala de aula aumentada e a sala invertida, desenvolvida por meio da descrição e explicação de pesquisas de outros autores. Obteve-se: Em primeiro lugar, na análise dos resultados da sua aplicação nas experiências de sala de aula invertida na área da saúde, os professores consideram que a interpretação do eletrocardiograma é um assunto complicado de aprender durante a realização de diagnósticos clínicos. A sala de aula invertida representa uma excelente alternativa para melhorar o processo de ensino-aprendizagem em medicina; Em segundo lugar, as experiências de aplicação de sala de aula invertida em que aparece um efeito de aprendizagem de alta velocidade que se reflete na resolução de exercícios e na aquisição de novas estratégias; Terceiro, há uma melhoria na frequência dos alunos em uma sala de aula virtualizada; Quarto, o clima produtivo de aprendizagem na sala de aula invertida está positivamente correlacionado com as percepções de aprendizagem em cirurgia na Universidad de la Sabana, Chía, Colômbia. Conclui-se que a sala de aula aumentada e a sala de aula invertida, assim como o conectivismo e a ubiqüidade, são os novos desafios da 
educação universitária que requerem adequada utilização e aplicação na formação de novos profissionais que o mundo global exige.

\section{Palavras chave}

Sala de aula aumentada, sala de aula invertida, conectivismo e onipresença.

\section{Introducción}

Los avances científicos y tecnológicos, así como el desarrollo de la psicopedagogía, la neuropsicología vinculados a los modelos constructivistas y conectivistas. El avance en el desarrollo de los sistemas de gestión de aprendizajes o plataformas virtuales, ofrecen una gran oportunidad la incorporación de aula aumentada y aula invertida en la formación de mentes humanas en este contexto global. Cavero Almenara, Leiva Olivencia, Moreno Martínez, Barroso Osuna, \& López Meneses (2016).

Aula aumentada y aula invertida, conectivismo y aprendizaje ubicuo en el área educativa son los nuevos retos en educación superior universitaria, requiere urgente atención por parte de los profesionales que ejercen la docente en las universidades.

Rey Tarazona \& Guevara Mejía (2021), Gabriela de la Cruz ha hecho referencia a la igualdad y equidad, las brechas en lugar de reducirse parecen ampliarse por las tendencias de la tecnología en la educación. Los docentes están en la obligación de diseñar nuevas estrategias de enseñanza para unos jóvenes nacidos en la hiperconectividad. De lo contrario la inadecuada aplicación de las TIC en el aula, la resistencia o rechazo por parte de los docentes incrementan las brechas digitales entre los estudiantes. Una concepción errónea de las TIC como elemento que favorece la inclusión social y educativa; repercute de manera negativa en estudiantes que se encuentran en riesgo de exclusión social, con menor dominio de tecnologías o que no cuenten con recursos digitales para la interacción.

Peinado Rocamora (2018, p. 183), en la clase invertida aparece un efecto de aprendizaje de gran velocidad reflejada en la resolución de ejercicios y adquisición de nuevas destrezas y procesos matemáticos. Por tal motivo, se tiene la finalidad de conocer a profundidad sobre aula aumentada y aula invertida. 


\section{Marco Teórico}

\subsection{Aula aumentada}

Cecilia Sagol (2021), afirma que el aula aumentada es el espacio virtual que complementa la clase real donde se puede generar complejidad de saberes lo que no se puede hacer en el aula real. Los objetos de aprendizaje de un aula aumentada se pueden publicar en una plataforma virtual o en el sitio de una red social virtual (Moodle, Google Classroom, Gogle Drive, bloq, grupo de Facebook, biblioteca virtual, youtube). Estos objetos de aprendizaje forman parte entre guías de aprendizaje, videos creados por el docente o estudiante, fotos, archivos en formato documentos, hojas de cálculos y en pdf donde es posible la participación de la comunidad educativa que ayuda a potenciar el aprendizaje. Cabe destacar que en un aula aumentada se incorpora temas de ciencia, arte, política, empresa, el mundo de la tecnología, la programación de aplicaciones, entre otros, por medio de la aplicación de estrategias de aprendizaje previamente evaluados y su posterior publicación de conocimientos o experiencias, con aceptación de las críticas que contribuyen a renovar e innovar el recurso para uso global.

El aula aumentada ayuda a crear tráfico de contenidos digitales, exige a trabajar con archivos. Los temas no se dicen, ni se explican en pizarra, sino, se convierten en archivos digitales que facilita la actualización, innovación y publicación de contenidos a gran escala. Sagol, Aulas aumentadas, lo mejor de los dos mundos (2021). Un aula aumentada debería estar vinculado indirectamente a temas del contexto social, a las necesidades o problemas de la población.

\subsection{Aula invertida}

Pérez Tornero y Tejedor (2016), universidades como el MIT (Instituto Tecnológico de Massachusetts) desde el año 2000, han incorporado y ofrecido abiertamente materiales de clase que se encuentran reconocidos en Internet. Asimismo, prestigiosas universidades del mundo han diseñado y ofertado por medio de los MOOC - Massive Open Online Course (Curso abierto en línea) a través del uso de herramientas tecnológicas de webinar, flipped 
classroom, learning analitycs, redes sociales, blogs, wikes, entre otros, certificaciones de aprendizajes después del primer gran éxito de 2012 del curso de Inteligencia Artificial ofrecido por la Universidad de Stanford y la reciente metodología de flipped classroom desarrollada por dos profesores norteamericanos de preparatoria y que varias universidades del mundo lo viene utilizando.

Berenguer Albaladejo, Acerca de la utilidad del aula invertida o flipped classroom (2019), la metodología Flipped Classroom o aprendizaje inverso, llamada también aprendizaje al revés o clase invertida, fue utilizado por dos profesores de la Universidad de Colorado en Estados Unidos, entre ellos Jonathan Bergmann y Aaron Sams, que utilizaron un software que permitía grabar presentaciones en Power Point y que comenzaron a publicar lecciones en internet para estudiantes que no podían asistir a clases de manera presencial. A partir de esta experiencia propusieron que los demás docentes grabasen contenidos para que los estudiantes lo visualizaran antes de cada clase. De este modo se invertían las actividades, generándose aprendizajes activos, colaborativos y efectivos en los estudiantes con materiales variados, atractivos y de buena calidad. Los materiales audiovisuales creados con carácter original, propio, breve, claro y conciso con propósitos de captar la atención de los estudiantes que posteriormente fueron ubicados en YouTube.

Cedeño Escobar \& Vigueras Moreno (2020), Flipped Classroom, aula invertida, aula volteada o aula inversa, es una estrategia didáctica, una metodología se caracteriza por una forma de enseñar, aporta mayor énfasis a la práctica. Su filosofía radica en que el estudiante aprende en cualquier lugar y practica en la clase, se adapta a los postulados del conectivismo por lo cual tiene gran aceptación en la empresa, negocio o educación.

Aguilera Ruiz, Manzano León, Martínez Moreno, Lozano Segura, y Casiano Yanicelli (2017), su importancia radica en que los contenidos son entregados de manera oportuna a los estudiantes por medio de una plataforma virtual para que sean estudiados la teoría y los conceptos, a fin de dedicar mayor tiempo a la práctica en las clases. La potencialidad de esta estrategia radica en que el tiempo invertido en explicar la materia de manera 
magistral queda de lado al trabajo que realiza el estudiante con la explicación estratégica en grabaciones de vídeo. Estos materiales son estudiados cuantas veces sea necesario, incluso puede consultar al docente y a sus compañeros a través de las redes sociales virtuales.

\subsection{Sistema de Gestión del Aprendizaje Online (LMS) para Aula Aumentada}

Wikipedia (2019), considera que existen distintas opciones de plataformas LMS en el mercado. Sin embargo, todos los sistemas ofrecen características similares de funcionalidad. El primer anuncio del LMS fue a fines de la década de 1990 y el primer LMS se utilizó en el sector de la educación superior. Se diseñaron para identificar las brechas de capacitación y aprendizaje. Surgió directamente de aprendizaje electrónico (e-Learning), hoy en día se centran en el mercado corporativo y que constituyen el segmento más grande del mercado de sistemas de aprendizaje.

Para el estudio de esta investigación, se consideró el sistema de gestión del aprendizaje Google Classroom por su sencillez, velocidad de acceso y servicio de almacenamiento en la nube.

2.3.1 Google Classroom: Guevara Maldonado, Magaña Domínguez, \& Picasso Hinojosa (2019), Geoogle Classroom, es una plataforma virtual educativa gratuita de blended learning, es decir aprendizaje semipresencial. Forma parte de la Suite de Google Apps for Education. Fue lanzada el 12 de agosto de 2014. El 15 de marzo de 2017, Classroom estaba disponible para cuentas personales de Google, en particular para las cuentas estándar de Gmail, se encuentra disponible en 42 idiomas. Entre sus funciones está simplificar y distribuir tareas, así como evaluar contenidos. Permite la creación de aulas virtuales dentro de una misma institución educativa, facilitando el trabajo entre los miembros de la comunidad académica.

Guevara Maldonado, Magaña Domínguez, \& Picasso Hinojosa (2019), Google Classroom, es una aplicación gratuita que forma parte del programa disponible para la comunidad universitaria Google Apps for Education (GAE). A través de Google Classroom 
se pueden utilizar las aplicaciones: Google Documents, Google Drive, Google Forms y Google Calendar, permitiendo así mayor efectividad y eficiencia. Esta herramienta tecnológica permite crear un aula virtual, donde se puede crear asignaciones, pruebas, distribuir lecturas, videos, tareas, crear foro de discusión, entre otras. Google Classroom permite ahorrar tiempo, organizar clases y comunicarse con los estudiantes. Es intuitivo y funcionable en cualquier dispositivo móvil. Atractivo para los estudiantes, su plataforma web se asemeja a una red social, como Facebook, con un muro, donde aparecen las asignaciones, comentarios del profesor y estudiantes, fechas importantes y anuncios. Por tanto, Google Classroom es realmente una aplicación de enseñanza enfocada en la interacción social.

\subsection{El Conectivismo}

Anna Beatriz (2021), George Siemens y Stephen Downes, autores del término conectivismo y e-Learning. Una teoría de aprendizaje para la era digital. Se fundamenta en la teoría del caos que indica la existencia de los hechos; el reto del aprendiz es organizar los modelos que supuestamente no son perceptibles. La teoría del caos como ciencia se conecta del todo y con el todo (ecología del conocimiento) y para aprender en la economía del conocimiento, se requiere tener la capacidad de efectuar conexiones entre fuentes de información y crear modelos de información relevantes. El conectivismo significa integración de principios explorados por la teoría del caos, redes informáticas, complejidad y auto organización. El aprendizaje se realiza en entornos indefinidos, puede establecerse fuera de uno mismo en una organización o en una base de datos, conectados a un conjunto de información especializada que hace posible profundizar el conocimiento.

Zurina Lestayo y Hernández Cáceres (2021), la Teoría del Caos estudia a ciertos tipos de sistemas dinámicos que no son lineales y a sistemas complejos muy sensibles a variaciones en condiciones iniciales. Las intensidades leves que se producen en la variación pueden generar grandes diferencias en el comportamiento de una situación que podría predecir el futuro; por lo que su comportamiento puede ser completamente determinado 
conociendo sus condiciones iniciales. El Caos puede analizarse en tiempo (sistemas dinámicos) y espacio (fractales). Su aparición ha generado un cambio del paradigma científico tradicional y posibilita encontrar el orden en el desorden. Más que una teoría, el Caos se ha convertido en un método, un modelo, una forma de generar conocimientos científicos en el área de los sistemas complejos.

\section{Figura 1}

\section{Esquema del conectivismo.}

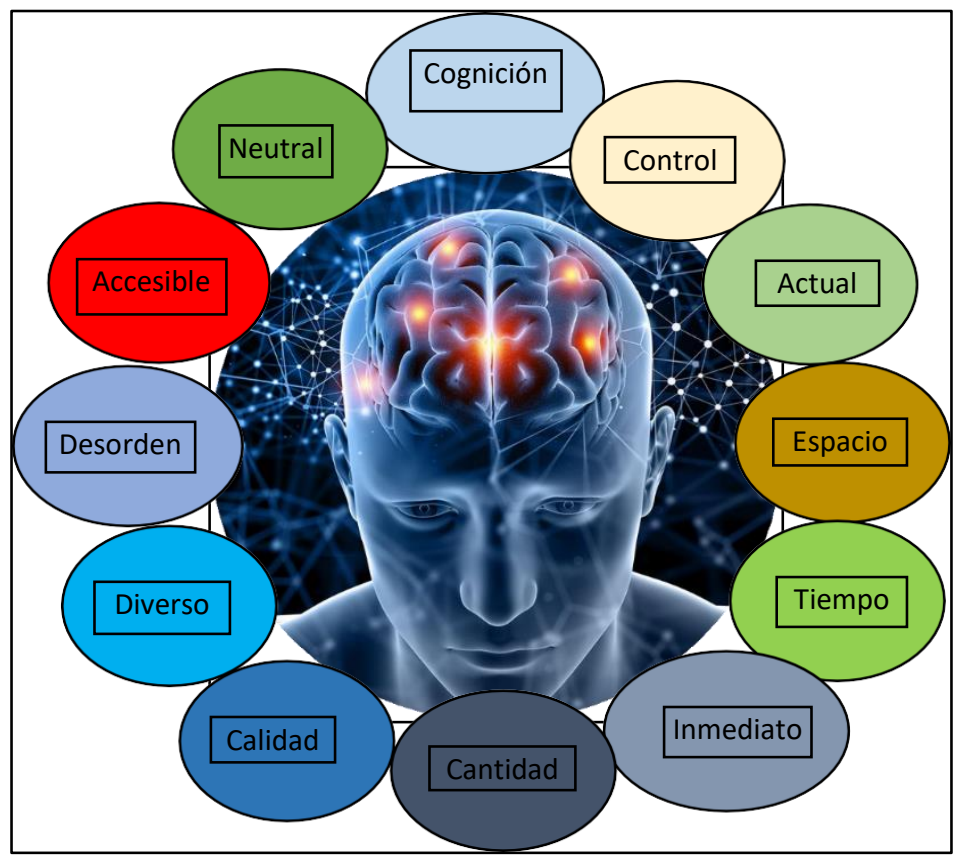

Nota: Conferencia conectivismo. Adecuación propia.

El conectivismo intenta explicar cómo aprendemos, integra y abarca aspectos de la vida implicados en la gestión y liderazgo, medios, noticias, gestión del conocimiento personal y organizacional, diseño de entornos de aprendizaje y aprendizaje en entornos complejos por las TIC que son necesarios para formar mentes humanas con actitudes de aprendizajes permanentes y cambiantes.

\subsubsection{Principios de la teoría del conectivismo:}


- El aprendizaje y el conocimiento se fundamentan en una diversidad de conceptos.

- El aprendizaje es un proceso de conexión entre nodos fuentes de información especializada y actualizada.

- El aprendizaje puede residir en dispositivos no humanos.

- La capacidad de conocer más es más importante de lo que sabes actualmente. El aprendizaje continuo requiere fortalecer y mantener las conexiones.

- La habilidad para ver conexiones entre campos, ideas y conceptos es una aptitud de mucha importancia.

- La circulación con conocimientos actualizados es el objetivo de todas las actividades de aprendizaje conectivistas.

- La decisión asumida, se convierte de inmediato en un proceso de aprendizaje. La elección de qué aprender y el significado de la información entrante son vistos mediante lentes de una realidad cambiada. Un resultado que en ese momento es correcto, puede variar debido a las alteraciones en el clima de la información.

Heredia Escorza y Sánchez Aradillas (2020), la teoría del Conectivismo, indica que se aprende haciendo conexiones con otras personas, ideas o conceptos. Las conexiones actuales son dinámicas y cambian rápido de acuerdo con los intereses y necesidades de los usuarios. Se fundamenta en el aprendizaje socio cultural, desarrollo próximo de Vygostky y la teoría de la actividad Engestrom, toda actividad se genera dentro de un contexto y para entender el significado es necesario conocerlo. Sobre el concepto de ecología de conocimiento refiere a un sistema educativo abierto, dinámico e interdependiente, diverso, adaptable y ágil. Creando posibilidades de un ambiente de aprendizaje significativo a los estudiantes que permita un desarrollo óptimo de posibilidad de construir su propio conocimiento.

\subsection{Aprendizaje Colaborativo Virtual}

Angulo Vilca (2021), un ambiente de aprendizaje debe tener espacios de colaboración y comunicación, apoyo para el aprendizaje colaborativo y facilitar la 
retroalimentación. Es importante discernir que no es la tecnología lo que genera aprendizaje, sino el uso adecuado de las tecnologías, las actividades programadas según el sílabo, un ambiente de aprendizaje, un público motivado y expectante. El aprendizaje colaborativo busca propiciar espacios que sean posibles el desarrollo de habilidades individuales y grupales a partir de la discusión de nuevos conceptos explorados entre los estudiantes. Incluye un conjunto de métodos de instrucción, entrenamiento apoyado con tecnología, estrategias para propiciar el desarrollo de habilidades mixtas (aprendizaje, desarrollo personal y social), mediante el desarrollo de cuestionarios, elaboración de guías de observación, exposiciones y desarrollar pruebas de conocimiento en Google Forms, Quizizz, Kahoot, documentos de Google, Meet y Zoom. Donde cada miembro del grupo es responsable de su aprendizaje y de los demás integrantes que son elementos básicos para la interdependencia positiva, interacción, intercambio de ideas y conocimiento entre el grupo de trabajo que permita conocer, compartir y ampliar la información que cada uno tiene de manera real y virtual.

\subsection{Aprendizaje Ubicuo (u-Learning)}

El concepto de aprendizaje ubicuo se originó en el marco de la informática o computación ubicua. Otras aproximaciones consideran a un tipo de aprendizaje móvil, que es un dominio del e-learning. Por otra parte, la influencia del enfoque de aprendizaje continuo y aprendizaje personalizado/adaptativo han establecido su definición más moderna. Desde el punto de vista del u-learning como un tipo de aprendizaje móvil, se describe como un aprendizaje que permite acceder a diversos entornos, contextos y situaciones, y que puede utilizar una mayor conciencia del contexto para proporcionar la mayoría de los contenidos que se adaptan a los estudiantes. No obstante, hay autores que señalan que el aprendizaje ubicuo no es un tipo de m-learning, sino que se trata de un concepto más profundo que representa su evolución, ya que el u-learning intenta aprovechar los diferentes dispositivos móviles o fijos, distintos momentos y modalidades a través de su integración para el aprendizaje. Esta visión del aprendizaje ubicuo es 
resultado de la influencia de dos enfoques: el aprendizaje continuo y el aprendizaje personalizado/adaptativo.

El aprendizaje continuo no se apoya en la tecnología como componente esencial, sino en la importancia de unir las experiencias de los estudiantes dentro y fuera de clase, sin interrupciones. El aprendizaje continuo es un hábito mental del estudiante para conseguir un flujo continuo de aprendizaje en todos los contextos, y puede o no ser intervenido por la tecnología. En cuanto al aprendizaje personalizado/adaptativo es un proceso que recoge las características individuales y las necesidades de los estudiantes, para generar actividades de aprendizaje significativas y relevantes, las cuales constantemente son autoiniciadas. Este tipo de aprendizaje también puede apoyarse o no en las tecnologías. En el primer caso, la tecnología emite recomendaciones basadas en las preferencias de los estudiantes.

Con el desarrollo de la tecnología, el aprendizaje es cada vez más diferente; se desarrolla en cualquier momento a través de cualquier dispositivo tecnológico que permita una comunicación e interacción, posible de realizarse en múltiples lugares, incluso en aquellos donde jamás se hubiese imaginado, como el hogar, el trabajo, supermercado, en las cafeterías, en un viaje.

\section{Figura 2}

Contenido de u-Learning. 


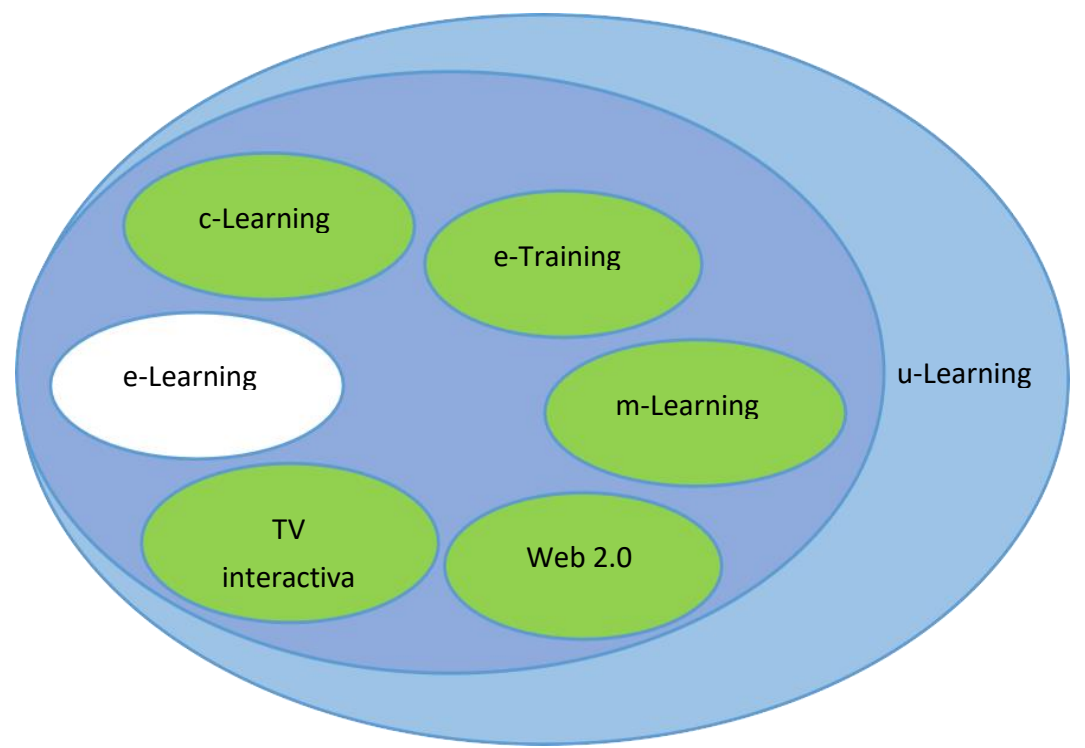

Nota: Rinaldi, 2011. Adecuación propia.

Tecnologías que integra el aprendizaje ubicuo (u-Learning):

- m-Learning (Mobile Learning). Acceso a servicios didácticos desde dispositivos móviles.

- c-Learning (Classroom Learning). Clases online, uso de chats, foros, grupos de discusión, simulaciones, estudio de casos, proyectos colaborativos, aprendizaje basado en proyectos.

- e-Training (Capacitaciones online). Entrenamiento o capacitación en línea a través de medios electrónicos. Se realiza en el ámbito empresarial, corporativo y organizacional mediante el uso de tecnologías y materiales en formatos digitales de auto estudio y autoaprendizaje.

- Televisión interactiva. Plataforma de televisión.

- Web 2.0. Web creada por usuarios, blogs, wikis, redes sociales.

El aprendizaje ubicuo (u-Learning) ofrece múltiples aprendizajes fuera del aula real por medio de ambientes distintos de la vida con apoyo de tecnología flexible, invisible y omnipresente que brinda información requerida en ese instante. 


\section{Materiales y métodos}

El trabajo metodológico de la investigación es de tipo descriptivo, explicativo y no experimental debido a que se revisó de manera sistemática y exhaustiva investigaciones por otros autores con la finalidad de analizar las evidencias de los resultados logrados sobre aula aumentada y aula invertida en educación superior universitaria; una metodología y un espacio de enseñanza y aprendizaje que exige la formación profesional en un contexto global.

\section{Resultados}

VivatAcademia revista de comunicación (2020), en el área de la Salud, los docentes consideran que la interpretación del electrocardiograma es un tema complicado de aprender durante la realización de los diagnósticos clínicos. Sin embargo, el aula invertida representa una excelente alternativa para mejorar el proceso de enseñanza aprendizaje en la Medicina, ya que permite analizar los recursos multimedia, libros electrónicos y lecturas digitales sobre el funcionamiento del corazón. En el salón de clases, los foros de discusión y el trabajo colaborativo permiten el rol activo de los estudiantes, motivo por lo cual se incrementó la satisfacción y desarrollo de las habilidades en los estudiantes. En Medicina Humana, el aula invertida facilita la participación de los estudiantes, ya que observan los videos y analizan las lecturas en la plataforma antes de la clase. La resolución de exámenes en línea ayudó a mejorar el rendimiento académico.

Peinado Rocamora (2018), un buen control teórico y la consecuente adquisición de herramientas muy útiles para la resolución de ejercicios prácticos, dota al alumnado de mayores aptitudes de capacidad reflexiva y de rectificación ante los errores producidos. En muchas fases de las experiencias el estudiantado asumió erróneamente que la teoría no es importante y que si se consiguen realizar los ejercicios con facilidad es suficiente. En la clase invertida aparece un efecto de aprendizaje de gran velocidad reflejada en la resolución de ejercicios y adquisición de nuevas destrezas y procesos matemáticos. Los 
procesos de planteamiento o cálculo, que tuvieron dificultades en el primer tratamiento, se resolvieron con facilidad los mismos ejercicios a partir de un segundo procedimiento. Con respecto a la asistencia registrada, cabe indicar que, su control e historial, es uno de los parámetros claves para la selección y seguimiento de los estudiantes dentro del curso. En el primer trimestre, se aplicó la metodología magistral, motivo por el cual el número de faltas por horas de clase es mayor. Según el historial de inasistencia en todos los cursos se incrementó en el segundo y tercer trimestre de cada curso. Sin embargo, con la aplicación del aula invertida, mejoró considerablemente la asistencia a clases de los estudiantes.

Peinado Rocamora (2018), la clase invertida supone una ganancia de tiempo importante dejando espacio para la resolución de ejercicios y para otras actividades en un sistema de gestión del aprendizaje. Si el estudiante que tiene dificultades a la hora de afrontar la resolución de ejercicios matemáticos encuentra ayuda en sus compañeros. La formación de grupos, parejas o tríos como máximo, contribuye a formar la unidad de aprendizaje más efectiva en la red. Los contenidos de naturaleza estrictamente teóricos, aquellos que requieren memorización, constituyen mayor tiempo para ser estudiados y repasados. Respecto a los recursos utilizados se comprueba que el vídeo es el más valorado, pero cuando su duración es más de diez minutos se considera como largo. Además, los videos necesitan ser complementados en clase para aclarar o ampliar algunas cuestiones que el vídeo no puede resolver.

Dominguez, Sanabria, y Sierra (2018), el modelo teórico demostró un buen ajuste estadístico con los datos empíricos. A partir de estos resultados se encontró que el clima de aprendizaje del aula invertida en cirugía en la Universidad de la Sabana, Chía, Colombia. Se relaciona positivamente con percepciones de aprendizaje, mediado por las habilidades académicas de los estudiantes, teniendo los resultados ( $\beta=0,35$; IC 95\% 0,26-0,43; $p<$ $0,001)$ y el papel de los profesores ( $\beta=0,31$; IC 95\% 0,23-0,39; $p<0,001)$. El clima productivo de aprendizaje en el aula invertida se correlaciona positivamente con las percepciones de aprendizaje en cirugía. 
IC: intervalo de confianza; $\beta$ coeficientes; P Hipótesis de correlación.

\section{Discusión}

VivatAcademia revista de comunicación (2020), la interpretación del electrocardiograma es un tema difícil de aprender por parte de los estudiantes durante la realización de los diagnósticos clínicos. Los recursos multimedia, los libros electrónicos y lecturas digitales sobre el funcionamiento del corazón virtualizados en una plataforma virtual, ayudan a mejorar el proceso de enseñanza aprendizaje en Medicina; el aula invertida facilita la participación de los estudiantes, la observación de videos y análisis de lecturas antes de la clase y la resolución de exámenes en línea contribuye en la mejora del rendimiento académico de los estudiantes.

Peinado Rocamora (2018, p. 183), en muchas fases de las experiencias el estudiantado asumió erróneamente que la teoría no es importante y que si se consiguen realizar los ejercicios con facilidad es suficiente. En la clase invertida aparece un efecto de aprendizaje de gran velocidad reflejada en la resolución de ejercicios y adquisición de nuevas destrezas y procesos matemáticos. Con respecto a la asistencia registrada, cabe indicar que, su control e historial, es uno de los parámetros claves para la selección y seguimiento de los estudiantes dentro del curso. En el primer trimestre, se aplicó la metodología magistral, motivo por lo que el número de faltas por horas de clase es mayor. En el segundo y tercer trimestre la inasistencia en todos los cursos se incrementó. Sin embargo, con la aplicación del aula invertida, mejoró considerablemente la asistencia a clases de los estudiantes.

La clase invertida supone una ganancia de tiempo importante dejando espacio para la resolución de ejercicios y para otras actividades en una plataforma. Si el estudiante que tiene dificultades a la hora de afrontar la resolución de ejercicios matemáticos encuentra ayuda de sus compañeros. La formación de grupos, parejas o tríos contribuyen a formar la unidad de aprendizaje más efectiva en la red. Los contenidos de naturaleza teórica, aquellos que requieren memorización, requieren de más horas para ser estudiados y repasados. 
Respecto a los recursos utilizados se comprueba que el vídeo es el más valorado, pero cuando su duración es más de diez minutos es considerado inadecuado. De manera que los videos necesitan aclarar o ampliar algunas cuestiones que no puede resolver.

Dominguez, Sanabria, y Sierra (2018), el clima productivo de aprendizaje en el aula invertida se correlaciona de manera positiva con las percepciones de aprendizaje en cirugía en la Universidad de la Sabana, Chía, Colombia.

\section{Conclusiones}

La realizó la revisión bibliográfica es de manera sistemática y exhaustiva de otros autores, con la finalidad de analizar las evidencias de los resultados logrados sobre aula aumentada y aula invertida en la educación superior universitaria.

Aula aumentada y aula invertida son una nueva forma de trabajo de enseñanza y aprendizaje que demandan las exigencias actuales para formar mentes humanas con competencias básicas necesarias para vivir y progresar en la sociedad, vinculadas con la convivencia, la comunicación y el procesamiento de información. Las competencias genéricas aquellas referidas a las profesiones, como gestión de recursos, trabajo en equipo, gestión de la información, resolución de problemas o planificación. Las competencias específicas las que determinan la profesión obligatorias e inevitables para obtener una cualificación.

La tecnología es un aliado en la empresa, en el negocio y en la labor docente que requiere adaptación a los avances científicos y tecnológicos de forma permanente. El impacto de las herramientas colaborativas como Google Classroom y conectivismo destacan su importancia por la sencillez que presenta para la implementación y aplicación de aula aumentada y aula invertida en cualquier nivel educativo.

Cabe mencionar que la inadecuada aplicación de las TIC en el aula, la resistencia o rechazo por parte de los docentes, incrementan las brechas digitales entre los estudiantes. De la misma forma, una concepción errónea de las Tecnologías de 
información y comunicación favorece de manera negativa a estudiantes en riesgo de exclusión social o con menor formación tecnológica o que cuenta con menores recursos digitales.

\section{Agradecimientos}

A los autores de las teorías que sirvieron de base científica del marco teórico y los resultados obtenidos permitieron orientar y corroborar los resultados sobre aula aumentada y aula invertida en la formación de estudiantes universitarios.

\section{Referencias}

Aguilera-Ruiz, C., Manzano-León, A., Martínez-Moreno, I., Lozano-Segura, M., \& CasianoYanicelli, C. (2017). EL MODELO FLIPPED CLASSROOM. International Journal of Developmental and Educational Psychology, pp. 261-266. doi:https://doi.org/10.17060/ijodaep.2017.n1.v4.1055

Angulo-Vilca, P. E. (2021). El aprendizaje colaborativo virtual para la enseñanza de la matemática. Revista Científica. Dominio de las Ciencias, pp. 253-267. doi:http://dx.doi.org/10.23857/dc.v7i1.1703

Anna-Beatriz, P. (2021). Conectivismo y diseño instruccional: Ecología de Aprendizajes para la Universidad del Siglo XXI en México. Márgenes, Revista de Educación de la Universidad de Málaga, 4-20. doi:https://doi.org/10.24310/mgnmar.v2i1.9349

Berenguer-Albaladejo, C. (2019). Acerca de la utilidad del aula invertida o flipped classroom. Obtenido de https://web.ua.es

Cavero-Almenara, J., Leiva-Olivencia, J. J., Moreno-Martínez, N. M., Barroso-Osuna, J., \& López-Meneses, E. (2016). Realidad Aumentada y Educación. Barcelona: Octa Edro. Obtenido de https://books.google.com.pe/books?id=OgilDwAAQBAJ\&printsec=frontcover \&dq= que+es+el+aula+aumentada\&hl=es-

419\&sa=X\&redir_esc=y\#v=onepage \&q=que\%20es\%20el\%20aula\%20aumentada\&f =false

Cedeño-Escobar, M. R., \& Vigueras-Moreno, J. A. (2020). Aula invertida una estrategia motivadora de enseñanza para estudiantes de educación general básica. Revista científica. Dominio de las ciencias, pp. 878-897.

doi:http://dx.doi.org/10.23857/dc.v6i3.1323 
Dominguez, L. C., Sanabria, Á. E., \& Sierra, D. O. (Noviembre de 2018). El clima productivo en cirugía: ¿una condición para el aprendizaje en el aula invertida? El Sevier, 7. Obtenido de https://www.sciencedirect.com/science/article/pii/S1575181317301663

Guevara-Maldonado, L. A., Magaña-Domínguez, E. A., \& Picasso-Hinojosa, A. L. (2019). EL USO DE GOOGLE CLASSROOM COMO APOYO PARA EL DOCENTE.

Heredia-Escorza,Y.,\& Sánchez-Aradillas, A. L. (2020). Teorías del Aprendizaje en el Contexto Educativo. Editorial Digital del Tecnológico de Monterrey, 2020 M07 2

Peinado-Rocamora, D. P. (2018). La clase invertida: una experiencia con alumnos con dificultades de aprendizaje. Universidad de Murcia. Escuela Internacional de Doctorado.

Pérez-Tornero, J. M., \& Tejedor, S. (01 de 08 de 2016). Ideas para aprender a aprender. Manual de Innovación Educativa y Tecnológica. Obtenido de https://books.google.com.pe/books?id=51PeDQAAQBAJ\&pg=PT35\&dq=como+utili zan+classroom+las+universidades+norteamericanas\&hl=es419\&sa=X\&ved=0ahUKEwjtkLOH4avpAhVFhuAKHXssDxgQ6AEIKDAA\#v=onepage \& q=como\%20utilizan\%20classroom\%20las\%20universidades\%20norteameric

Rey-Tarazona, C. I., \& Guevara Mejía, P. A. (04 de Junio de 2021). Resignificar el rol del docente con el uso de las tecnologías en la educación. Obtenido de https://repositorio.uniagustiniana.edu.co/handle/123456789/1727

Sagol, C. (25 de Agosto de 2021). Aulas aumentadas, lo mejor de los dos mundos. Obtenido de http://mjpa-educa.blogspot.com/2013/08/aulas-aumentadas-lomejor-de-los-dos.html

VivatAcademia revista de comunicación. (2020). Obtenido de http://www.vivatacademia.net/index.php/vivat/article/view/1238

Wikipedia. (12 de Diciembre de 2019). ¿Qué es un LMS y por qué es esencial para el aprendizaje corporativo? Obtenido de https://www.ispring.es/blog/what-is-Ims

Zurina-Lestayo, O., \& Hernández-Cáceres, J. L. (2021). Cultura del caos. Revista Cubana de Informática Médica. Obtenido de Zurina-Lestayo, O., \& Hernández-Cáceres, J. L. (2021). Cultura del caos. Revista Cubana de Informática Médica. 